Colman, A. M., \& Stirk, J. A. (1999). Singleton bias and lexicographic preferences among equally valued alternatives. Journal of Economic Behavior and Organization, 40, 337-351.

Singleton Bias and Lexicographic Preferences

Among Equally Valued Alternatives

Andrew M. Colman and Jonathan A. Stirk

University of Leicester, U.K.

\begin{abstract}
Two experiments examined a form of singleton bias deducible from lexicographic choice and Tversky's theory of elimination by aspects. In Experiment 1, 100 decision makers who chose from a set of job applicants defined by equal numbers of equally important qualifications tended to ignore the singleton defined by possession of a relevant attribute but tended to rank-order the attributes as predicted by lexicographic choice theory and showed various forms of singleton bias. In Experiment 2, 100 decision makers who chose from sets of unspecified alternatives, universities, and houses/apartments defined by attributes that they had individually rated as equally important ignored the attribute-defined singleton in every alternative set but manifested a different singleton bias in favor of the middle option of the on-screen display.
\end{abstract}

JEL classification code: C91

Key words: elimination by aspects, framing, lexicographic ordering, multi-attribute decision making, singleton bias 


\section{Singleton Bias and Lexicographic Preferences \\ Among Equally Valued Alternatives}

Everyday decisions are often made from sets of alternatives that vary on a number of attributes, aspects, or criteria. For example, choosing a house may involve comparing alternatives according to price, location, and number of rooms; hiring an employee may involve comparing candidates according to multiple qualifications; and selecting a marriage partner may involve comparing suitors on attributes such as physical attractiveness, intelligence, and sense of humor. According to the standard linear additive valuemaximization model of multi-attribute choice, the decision maker simply weights the attributes according to their perceived importance, then sums the weights, then finally chooses the alternative with the highest aggregate weight. This model appears to predict multi-attribute choices robustly in certain circumstances (Dawes, 1979; Slovic and Lichtenstein, 1971).

There are reasons to doubt that the linear value-maximization model accurately reflects the behavior of decision makers in complex multi-attribute choices, however. The following counterexample adapted from Tversky (1972, p. 283) is typical. Suppose a decision maker has to choose between two travel agencies $A_{1}$ and $A_{2}$ offering tours to two different destinations $D_{1}$ (Africa) and $D_{2}$ (the Far East). Agency $A_{1}$ offers only $D_{1}$, whereas agency $A_{2}$ offers both $D_{1}$ and $D_{2}$. The decision maker is equally attracted by Africa and the Far East and is indifferent between the travel agencies. According to the linear value-maximization model, there are three alternatives represented by feasible combinations of travel agency and destination, $A_{1} D_{1}, A_{2} D_{1}, A_{2} D_{2}$, and because they are equally valued, their probabilities of being chosen are equal. But it is intuitively obvious that most human decision makers would in practice choose first between destinations $D_{1}$ and $D_{2}$ (Africa and the Far East) and only then between travel agencies $A_{1}$ and $A_{2}$, and consequently the probabilities $P$ of choosing 
each of the three alternatives are $P\left(A_{1} D_{1}\right)=P\left(D_{1}\right) P\left(A_{1}\right)=(1 / 2)(1 / 2)=1 / 4, P\left(A_{2} D_{1}\right)=$ $P\left(D_{1}\right) P\left(A_{2}\right)(1 / 2)(1 / 2)=1 / 4$, and $P\left(A_{2} D_{2}\right)=P\left(D_{2}\right)=1 / 2$. Thus, one of the alternatives has twice the probability of being chosen that either of the others has. Empirical evidence suggests that human decision makers commonly use such lexicographic decision strategies that involve considering the attributes in order of priority (Ford, Schmitt, Schechtman, Hults, and Doherty, 1989; Tversky and Sattah, 1979; Westenberg and Koele, 1994). This implies that the "robust beauty" (Dawes, 1979) of the linear value-maximization model may be spurious and may not reflect the true psychological processes underlying multi-attribute decision making.

The theory of lexicographic preferences was first formalized by Georgescu-Roegen (1954); then Encarnación (1964) explored a class of $L^{*}$ lexicographic orderings that model Simon's (1957, pp. 241-273) bounded-rationality concept of satisficing -- choosing an alternative that is satisfactory or that suffices, rather than seeking to optimize. Day (1971, 1996) developed the theory further by establishing the continuity and convexity properties of $L^{*}$ choices and the existence of competitive equilibria in an economy of $L^{*}$ agents. According to the model, a decision maker first considers the alternatives that satisfice the most important or top-ranked attribute, then, restricting attention to alternatives that attain this level, considers the alternatives that satisfice the second-ranked attribute, and so on until the decision process focuses on a single alternative or a set of alternatives among which the decision maker is indifferent.

Suppose that a decision maker chooses a single alternative from a set $A=\{x, y, \ldots\}$ and that each alternative $x$ is represented by a vector $(x)=\left(x_{1}, x_{2}, \ldots\right)$, where $x_{i}$ is the perceived value of the ith attribute of alternative $x$, and the attributes are arranged in hierarchical order, with $i=1$ being the most important attribute that the decision maker considers first, $i=2$ the second most important attribute that the decision maker considers second, and so on. To avoid the complications that arise from attributes that exceed satisficing levels by significant 
margins and thus produce slack variables (in the linear programming sense), let us consider the straightforward case of attributes that are either present or absent and that satisfice if and only if present, so that $(x)$ is a zero-one vector with every component $x_{i} \in\{0,1\}$. Then $x_{1}>y_{1}$ implies that $x$ is lexicographically preferred to $y$, and in general $x_{1}=y_{1}, x_{2}=y_{2}, \ldots, x_{m}>y_{m}$ implies that $x$ is lexicographically preferred to $y$.

When choices are made from sets of two or more alternatives that are equally valued, decision makers' lexicographic preference orderings are revealed. Slovic (1975) had decision makers choose between gift packages of cash and coupons that they themselves had equated for value a week earlier (e.g., Package A: \$18 gift coupons and \$20 cash; Package B: \$32 gift coupons and $\$ x$ cash; $x$ set by the decision maker to equalize the value of the two packages). The decision makers also indicated which attribute (cash or coupons) they considered more important. According to the linear value-maximization model, because the two packages were equally valued, the decision makers should have chosen them equally often, but in fact 88 per cent chose the alternative that was higher on the attribute that they considered more important (usually the cash). In other words, most decision makers evidently used a lexicographic choice procedure that revealed their importance ranking of cash over coupons. Slovic replicated this finding in numerous different domains, including choices between college applicants, car tires, baseball players, and routes to work (see also Tversky, Sattah, and Slovic, 1988).

In choices among equally valued alternatives, an alternative has an increased probability of being chosen if it is perceived to possess an attribute that the other alternatives lack. We use the term singleton bias to denote a hypothesized tendency to prefer an alternative that is perceived as unique by virtue of an attribute that does not affect its desirability or attractiveness according to linear value-maximization theory. In Tversky's (1972) example of the travel agencies discussed above, among the three combinations of travel agencies and destinations $\left(A_{1} D_{1}, A_{2} D_{1}, A_{2} D_{2}\right)$, there are two unique attributes, $A_{1}$ and $D_{2}$. The alternative 
$A_{2} D_{2}$ that uniquely contains the destination $D_{2}$ (the Far East) has the highest probability of being chosen because destination is a more important attribute than travel agency and is therefore considered first. If travel agency were more important than destination, then $A_{1} D_{1}$, which uniquely contains $A_{1}$, would have the highest probability of being chosen. If we did not know that destination is more important than travel agency, we could infer it from the greater probability of $A_{2} D_{2}$ being chosen in an experimental test.

What happens under lexicographic choice when attributes are perceived as equally important? It is reasonable to assume that the decision maker chooses one of the attributes at random, eliminates alternatives that do not satisfice on that attribute, then chooses a second attribute at random, and so on. In these circumstances lexicographic choice results in singleton bias if a singleton is available. Suppose $A=\{x, y, z\}$, and the attributes distributed among the three alternatives are $\alpha, \beta, \gamma, \delta$, and $\varepsilon$ and are perceived by the decision maker as equally important. If $X=\{\alpha, \gamma, \varepsilon\}, Y=\{\beta, \gamma, \delta\}$, and $Z=\{\beta, \delta, \varepsilon\}$, then each alternative possesses exactly three equally important attributes, but alternative $x$ is a singleton inasmuch as it is the only one with a unique attribute $(\alpha)$, all other attributes being shared by two alternatives. Because the five attributes are equally important, we hypothesize that the decision maker considers them in a random order. The probability $P(x, A)$ of $x$ being chosen from the alternative set $A$ is equal to the probability of $\alpha$ being considered first, plus the probability of $\gamma$ being considered first and $\alpha$ or $\varepsilon$ second, plus the probability of $\varepsilon$ being considered first and $\alpha$ or $\gamma$ second. Hence $P(x, A)=(1 / 5)+(1 / 5)(1 / 2)+(1 / 5)(1 / 2)=.40$. However, by analogous reasoning $P(y, A)=P(z, A)=(1 / 5)(1 / 2)+(1 / 5)(1 / 2)+(1 / 5)(1 / 2)=$ .30. Table 1 shows how these choice probabilities are built up.

Table 1 about here

A stochastic version of lexicographic choice is incorporated in Tversky's (1972) 
influential theory of elimination by aspects (EBA). According to the theory, a choice is reached through an iterated series of eliminations. At each iteration, the decision maker selects an attribute, the probability of selection being proportional to the attribute's perceived importance, and eliminates all alternatives lacking that attribute, then selects the next most important attribute and proceeds in the same way, and so on until all but one of the alternatives have been eliminated. Formally, $P(x, A)$ is the probability of choosing alternative $x$ from the set $A$ of available alternatives, $P(x, A) \geq 0 \forall x \in A$ and $\Sigma_{x \in A} P(x, A)=1$. Each alternative $x$ is mapped to a finite set $X=\{\alpha, \beta, \ldots\}$ of attributes of $x$, with the interpretation that $x$ possesses the attribute $\alpha$ iff $\alpha \in X$. A non-negative number $u(\alpha)$ represents the utility or value of each attribute $\alpha$, and $A_{\alpha}$ is the set of alternatives in $A$ that possess the attribute $\alpha$. The following recursive formula defines the EBA model:

$$
P(x, A)=\frac{\sum_{\alpha \in X} u(\alpha) P\left(x, A_{\alpha}\right)}{\sum_{\beta \in A} u(\beta)},
$$

where at each recursive step attributes that are common to all the alternatives in the current set $A$ are excluded from both $X$ in the numerator and $A$ in the denominator. Equation 1 expresses the probability of choosing $x$ from $A$ as a weighted sum of the probabilities of choosing $x$ from various proper subsets of $A$ that contain each of the attributes $\alpha$, that is, from every $A_{\alpha}$ such that $\alpha \in X$. The weighting term $u(\alpha) / \Sigma u(\beta)$ represents the probability of selecting an attribute $\alpha$ from among all attributes $\beta$ in the current alternative set.

Applying Tversky's (1972) EBA model to the example discussed earlier in which $X=\{\alpha$, $\gamma, \varepsilon\}, Y=\{\beta, \gamma, \delta\}$, and $Z=\{\beta, \delta, \varepsilon\}$, because the five attributes are equally important to the decision maker, we can set $u(\alpha)=u(\beta) \forall \alpha \in X$ and $\beta \in A$ in Equation 1 and $u(\alpha) / \Sigma u(\beta)$ varies simply with the number of alternatives in A. Assuming random attribute selection, iterated application of Equation 1 reduces to standard lexicographic choice. By substituting in Equation 1, we find that $P(x, A)=.40, P(y, A)=.30$, and $P(z, A)=.30$ as before (see Table 1$)$. 
This is an instance of the principle first noted by Tversky (1972) that "the introduction of an additional alternative `hurts' similar alternatives more than dissimilar ones” (p. 296).

In consumer choices from sets of equally valued alternatives, irrelevant attributes are sometimes used to create meaningful differentiation (Aaker, 1991). A consumer commodity that is differentiated from its competitors by a unique but irrelevant attribute may be chosen significantly more often if its salience helps the consumer to simplify the decision by distinguishing it as an odd one out or a singleton. The standard example of an irrelevant attribute is the "flaked coffee crystals" or granules of a certain brand of instant coffee; but this granular attribute may not be entirely irrelevant because, even though granules and powder are the same once dissolved, the dry granules may be easier to handle without spilling. Carpenter, Glazer, and Nakamoto (1994) provided empirical evidence that consumers sometimes behave as though they value supposedly irrelevant attributes, even when they know that the attributes are really irrelevant. But as the example of the granulated coffee shows, it is difficult to be sure that such an attribute adds no subjective value, and in fact the second of Carpenter, Glazer, and Nakamoto's experiments showed that if the alternative with the putatively irrelevant attribute is also more expensive, then consumers tend to believe that the attribute must be relevant.

The experiments described in this article were designed to test the hypothesis of lexicographic choice leading to singleton bias and at the same time to circumvent the methodological problems of previous research, in which singletons were created by providing them with extra attributes that were supposedly irrelevant. Our experiments involve singletons that are unique, not by virtue of possessing irrelevant attributes that may turn out to have nonzero values for the decision makers, but through their possession of relevant attributes that the other alternatives lack. In Experiment 1, decision makers chose from among candidates for a job having identical numbers of relevant attributes, which the decision makers were instructed to treat as equally important, but one of the candidates had a 
unique relevant attribute that the others lacked, whereas the others shared each of their relevant attributes with another candidate. In Experiment 2, the decision makers chose from among sets of three alternatives defined by attributes that they had individually equated for importance, but once again the alternative set always included a singleton with a relevant attribute that the other alternatives lacked.

\section{Experiment 1}

\section{Method}

Participants. The participants were 100 undergraduate students at the University of Leicester, 77 female and 23 male, aged from 17 to 44 years $(M=21.90, S D=5.78)$. All participants were volunteers who received course credits in return for their participation.

Experimental design. Participants were assigned randomly to five groups. In each group they were presented with a specially designed job recruitment advertisement and three curricula vitae relating to candidates for the post. The advertisement specified five job requirements, which were described as "all equally important", and each curriculum vitae portrayed a candidate who fulfilled exactly three of these job requirements. Apart from the manipulation of the job requirements (explained below), the curricula vitae were kept as similar as possible.

The curricula vitae were constructed so that in every group, although each of the three candidates exhibited exactly three of the five qualifications specified in the job requirements, there was one designated qualification that was possessed by just one of the candidates, the other four designated qualifications being shared by two of the candidates. The details of the experimental design are shown in Table 2 , in which the first group is identical to the example of Table 1, and the other groups are simply permutations of the same basic structure.

Table 2 about here 
In each group there were three candidates, each of whom possessed three of the designated qualifications (arbitrarily numbered 1, 2, 3, 4, and 5), and so if these qualifications are equally weighted, then according to linear value-maximization theory the three candidates should have equal probabilities of being chosen. However, in Group 1 there was a singleton candidate $(A)$ who was the unique possessor of a designated attribute (qualification 1). Similarly, in Group 2 Candidate $A$ was a singleton through being the unique possessor of a different designated attribute (qualification 2), and so on for each group. The labelling and positioning of alternatives and attributes in Table 2 are for purposes of exposition only and were not communicated to the experimental participants. The design was fully balanced in the sense that in each group a different qualification was designated as the singleton and the remaining qualifications were equally represented.

Procedure. Participants were tested in small groups, up to eight at a time, but they worked entirely on their own, without any discussion or exchange of views. They were presented with the following written instructions:

You will be shown a job recruitment advertisement and a curriculum vitae (CV) from each of three candidates for the job. . . . Imagine that these are the only candidates who made it on to the short-list, and that you have to choose one of the three candidates immediately without conducting interviews or seeking any further information. Please indicate your choice in the space below and, if possible, briefly mention the reason for your decision. ...

Participants were then shown the following job recruitment advertisement for a "Market Development Specialist":

British United Shoe Machinery Ltd, the world's largest company manufacturing and 
marketing both machinery and materials for the footwear industry, wishes to appoint a Market Development Specialist to help with our machinery spare parts business. The successful candidate will be responsible for strengthening our market position, and responsibilities will encompass all elements of the marketing mix to create growth of our revenues and margins as well as market share. Preference will be given to candidates with the following, which are all equally important: formal qualifications in marketing, managerial experience, a knowledge of the footwear manufacturing industry, additional experience in a different engineering or manufacturing industry, and some foreign language capability.

Participants were then presented with three curricula vitae on separate sheets of paper, all together in no particular order, and they indicated their choices and "brief reasons for choice" on an answer sheet, together with information about their own gender and age.

\section{Results and Discussion}

The relative frequency of singleton choice across groups was 38/100, compared with an expected frequency under the null hypothesis of random choice of 33.33/100. The deviation is in the hypothesized direction, but the effect was non-significant: $\chi^{2}(1, N=100)=0.19, n s$. Separate analyses on a group-by-group basis (see Table 3) show that this finding masked a more complex pattern of results.

Table 3 about here

Choices deviated significantly from chance in two of the five groups. In Group 1, 70 per cent of decision makers chose the singleton candidate, compared to a chance expectation of 33.33 per cent; this result was highly significant: $\chi^{2}(2, n=20)=14.79, p<.001$, and the effect size, using Cohen's $(1988,1992)$ index $w$, was 0.86 , which is large. In that group, at 
least, the experimental hypothesis was corroborated by the observed choices. However, in Group 2, 85 per cent of decision makers chose Candidate $C$, who was not the singleton: $\chi^{2}(2$, $n=20)=24.09$, effect size $w=1.10$ (large). There were slight, though non-significant, deviations toward the singleton in Groups 3 and 4 and away from the singleton in Group 5.

The relative frequencies with which the designated qualifications appeared in the chosen candidates' CVs are shown in Table 4.

Table 4 about here

Qualification 3 (“a knowledge of the footwear manufacturing industry") was chosen most frequently, but if the summed frequencies are compared for goodness of fit with an expected uniform distribution (60 for each qualification), then the largest residual is associated with qualification 4 (“other manufacturing or engineering experience"), which was chosen most infrequently. Every decision maker contributed three data points (one for each qualification of the chosen candidate), hence the scores are not independent and differences cannot be tested for significance, but each triplet was chosen by a different decision maker, and these independent frequencies are shown in Table 5.

Table 5 about here

The marginal totals in the right-hand column of Table 5 were analyzed with a chi-square test, using expected frequencies based on the relative frequencies with which the combinations were represented in the total set of curricula vitae. The result was as follows: $\chi^{2}(9, N=100)=39.66, p<.001$, effect size $w=0.63$ (large). By far the most popular combination of qualifications was 1, 3, 5 ("some foreign language capability"; "knowledge of the footwear industry"; and "managerial experience"), and this combination was associated 
with the largest residual. This presumably reflects a culturally shared opinion among the participants that these are relatively important attributes, and it reveals a strong tendency to prioritize attributes, as predicted by lexicographic choice theory.

Qualitative analysis of reasons for choices. Decision makers were asked to "briefly mention the reason for your decision". Responses fell into a relatively small number of distinct categories shown in Table 6.

Table 6 about here

None of the participants mentioned the fact that one of the candidates was the unique possessor of a designated attribute. The most common reasons involved implicit underweighting or overweighting of one or more of the attributes that were supposed to be weighted equally. Almost two-thirds (103/160) of the reasons indicated unequal weightings, which could have led to nonrandom lexicographic orderings. The next most frequent reasons for choice involved non-designated and therefore supposedly irrelevant attributes or subsets of attributes, which accounted for just under one-fifth (28/160) of the reasons cited, and the inference of non-designated and hence irrelevant attributes or subsets of attributes not specifically mentioned in the curricula vitae, which accounted for just under 7 per cent $(11 / 160)$ of the reasons. Taken together, unequal weighting of attributes and use or inference of irrelevant attributes accounted for more than 88 per cent $(142 / 160)$ of the reasons for choice.

The singletons were apparently too well hidden for the decision makers to detect, at least consciously. But some of the reasons in Table 6 suggest other forms of singleton bias. Unequal weighting of designated attributes, possibly affecting lexicographic orderings, does not fall within our definition of singleton bias, because the adjusted weights may cause the chosen candidate to be the most attractive according to linear value-maximization theory. But 
the use of irrelevant (non-designated) attributes, and the inference of irrelevant attributes, are clear instances of singleton bias, and almost a quarter (39/160) of the reasons fell into these two categories. In other words, almost a quarter of the reasons for choice involved the identification of the chosen candidate as an odd one out by virtue of some property that should not have influenced the decisions according to linear value-maximization theory. If we ignore unequal weighting of attributes, more than two-thirds (39/57) of the reasons are interpretable as instances of singleton bias.

In Experiment 1 many decision makers were apparently unable to avoid prioritizing attributes. To expose this phenomenon more clearly, one approach would be to use alternatives framed as abstract decision tasks, stripped of all contextual information. This would reduce distortions arising from the preconceptions that decision makers bring to the task but would also involve a sacrifice of realism and ecological validity. Experiment 2 therefore included both an abstract and two lifelike decision frames. The most important innovation was the implementation, via an interactive computer interface, of a procedure to ensure that each decision maker was forced to choose between attributes that he or she had personally weighted equally. The purpose of this was to eliminate the difficulty that some decision makers evidently experienced in Experiment 1 of assigning equal weight to attributes that they did not consider equally important.

\section{Experiment 2}

\section{Method}

Participants. The participants were 100 undergraduate students at the University of Leicester, 79 female and 21 male, aged from 18 to 48 years $(M=22.26, S D=7.33)$. They were all volunteers and received course credits in return for their participation. They were recruited through posters advertising a "decision making experiment".

Experimental design. The participants were presented with three decision tasks involving the following contextual frames: choosing a university, choosing a house or apartment, and 
choosing an unspecified alternative in an abstract decision frame. The participants rated a set of potential attributes for importance and then made a choice from among three alternatives that were assembled from attributes that they themselves had equated for importance in the previous rating phase. The order of presentation of decision frames was counterbalanced, each of the six possible permutations of the three decision frames occurring with almost identical frequency subject to the constraint that the number of participants was not perfectly divisible by six. In every alternative set, exactly one of the alternatives was a singleton with a designated attribute that the other alternatives lacked, and the position of the singular attribute in the list of attributes was fully counterbalanced.

General procedure. Participants sat in front of computer monitors and followed a sequence of on-screen instructions that guided them in rating the importance of 25 attributes associated with each decision frame (except the abstract frame) and then choosing from among three alternatives constructed by the computer from five attributes that they had individually equated for importance. The participants rated the attributes and made their choices in their own time, which amounted to 20-30 minutes in most cases. After completing the task, they were thanked for their participation and debriefed.

Description of computer program. The computer program that controlled the on-screen information ensured that the decision makers were always constrained to choose from three alternatives defined by attributes that they had individually equated for importance. Except in the abstract decision frame, the program began by presenting an on-screen display of 25 potential attributes, and the decision maker was asked to rate each attribute on a scale from 1 ("not at all important") to 5 ("very important") in choosing a university or a house/apartment to rent. On the basis of these ratings the program selected five attributes that the decision maker had rated equally; with 25 potential attributes and a five-point rating scale, the existence of at least five equally rated attributes was guaranteed. The five equally rated attributes were used in the choice phase of the experiment to define the three available 
alternatives. The three alternatives each consisted of a list of five designated attributes, three of which the alternative did possess and two that it did not possess. Exactly one of the three was unique in possessing an attribute that the other two lacked. In the abstract decision frame, there was no rating of attributes, and the decision makers proceeded straight to the choice phase of the experiment. An example from one of the lifelike decision frames is shown in Table 7.

Table 7 about here

In Table 7, the singleton is House $A$ and the singleton position is first in the list of attributes of that alternative. Each of the three alternatives has exactly three of the attributes that the decision maker equated for importance in the previous rating phase. However, House $A$ is the only alternative that has an attribute that the other two alternatives lack (gas central heating). The position of the singular attribute was counterbalanced both as regards the alternative within which it appeared $(A, B$, or $C)$ and where it appeared in the list of five defining attributes. In the abstract decision frame, the choice was from alternatives $A, B$, and $C$ having or not having attributes labeled simply $V, W, X, Y$, and $Z$. The full counterbalanced design is shown in Table 8.

Table 8 about here

After recording their choices, the decision makers repeated the whole procedure for each of the other two decision frames, the order of presentation of the decision frames being fully counterbalanced, and they were then presented with a screen containing debriefing information.

Results and Discussion 
The singleton and non-singleton choices are displayed in Table 9, together with the random frequencies expected under the null hypothesis.

Table 9 about here

In all three decision frames, the observed choices deviated from the expected frequencies in the direction of singleton bias, although these deviations were non-significant. For the university frame, $\chi^{2}(1, N=100)=1.91, n s$; for the house/apartment decision frame, $\chi^{2}(1, N=$ $100)=0.29, n s ;$ and for the abstract decision frame, $\chi^{2}(1, N=100)=0.92, n s$.

A hierarchical log-linear analysis was carried out to determine whether the position of an alternative in the on-screen display (left, middle, or right) or the interaction between singleton status and on-screen position had a significant effect on the decision makers' choices. (In Experiment 1, the alternatives were presented all together on separate sheets of paper and the position variable was undefined.) After deleting the non-significant interaction effects, the on-screen position turned out to be statistically significant in all three frames: university frame, $\chi^{2}(1, N=100)=5.87, p<.05$, effect size $w=0.24$ (small); house/apartment frame, $\chi^{2}(1, N=100)=9.47, p<.01$, effect size $w=0.30$ (medium); and abstract frame, $\chi^{2}(1$, $N=100)=10.30, p<.01$, effect size $w=0.32$ (medium). In all three cases the largest residuals were associated with the middle position, indicating a significant tendency to choose the middle alternative in each case. In other words, the decision makers tended to resolve the balanced decision problem with a rather unsophisticated form of the singleton bias by introducing a positional attribute and choosing the middle alternative.

General Discussion

The experiments described in this article were designed to test the hypothesis that human decision makers choose according to lexicographic preference orders and therefore manifest a form of singleton bias deducible from lexicographic choice and Tversky's (1972) EBA 
model. Singleton bias was defined as a tendency to choose an alternative that is perceived as unique by virtue of a property that does not affect its desirability or attractiveness according to linear value-maximization theory. We circumvented a problem in the interpretation of Carpenter, Glazer, and Nakamoto's (1994) study of the irrelevant attribute heuristic, where putatively irrelevant attributes were in fact subjectively valued by decision makers. Our experiments focused on singleton bias in choices from equally valued alternatives without irrelevant attributes. The decision makers in both experiments were presented with sets of alternatives that were intended to be equally valued and that always included one alternative that was a singleton, not because of its possession of any supposedly irrelevant attribute, but through its unique possession of a relevant attribute, the other alternatives having only shared attributes.

In Experiment 1, choices deviated significantly from chance in two of the five groups. In one group the singleton alternative was chosen significantly more frequently than the other two, but in another group one of the non-singletons was chosen significantly more frequently. The first of these effects offers some corroboration of the experimental hypothesis, but data from the decision makers' self-reported reasons for choice suggest that the singularity was not salient and probably played no part in determining their choices. However, certain attributes and combinations of attributes were chosen significantly more frequently than others, suggesting a strong tendency to prioritize attributes. These findings are consistent with the hypothesis that human decision makers have a strong and perhaps irresistible propensity to arrange wants in hierarchies, as suggested by Georgescu-Roegen (1954). The results of Experiment 2 suggest, once again, that the decision makers failed to respond to the singletons that were built into the experimental design, even in the abstract decision frame that eliminated distracting information to make the singleton more conspicuous, but that many of them introduced a positional attribute as a tie-breaking device and chose the middle option. 
Although there is little evidence that the decision makers responded to the singletons that were deliberately introduced into the experiments, there is indirect evidence of lexicographic choice behavior and singleton bias. In Experiment 1, had the participants treated the designated attributes as equally important, and had they evaluated the alternatives solely according to the designated attributes, then lexicographic choice would have led to singleton bias. The qualitative data suggest that they did not in general regard the attributes as equally important and did not invariably confine their evaluations to the designated attributes.

The data shown in Tables 4 and 5 suggest that the importance weightings assigned to attributes and to combinations or subsets of attributes are largely comprehensible if interpreted as revealed lexicographic preferences. For example, it is clear from Table 4 that, in choosing a Market Development Specialist for the footwear industry, decision makers chose candidates with qualification 4 ("other manufacturing or engineering experience") far less frequently than candidates with other designated qualifications, and they chose candidates with qualification 3 ("a knowledge of the footwear manufacturing industry") far more frequently. These findings suggest that the decision makers in general failed to regard the attributes as equally important, and that it was lexicographic ordering that enabled them to identify uniquely qualified candidates. This is not difficult to understand: "other manufacturing or engineering experience" is vague and nonspecific, and "a knowledge of the footwear manufacturing industry" seems as relevant as can be for a senior post in "British United Shoe Machinery Ltd, the world's largest company manufacturing and marketing both machinery and materials for the footwear industry", as it was described in the instructions. Also, Table 5 shows that candidates with one particular combination of qualifications ("some foreign language capability", "knowledge of the footwear industry", and "managerial experience") were chosen far more frequently (almost twice as frequently) than candidates with any other combination of qualifications. It is reasonable to infer that this combination was (perhaps understandably) weighted more heavily than any other and that it enabled 
singleton candidates to be identified. Thus the decision makers revealed their lexicographic preferences in choosing among the equally valued alternatives.

Table 6 shows that many of the decision makers' self-reported reasons for choice involved considerations of non-designated attributes that were supposed to have zero weights: 28 of the 160 reasons fell into this category, and a further 11 involved inferences of irrelevant attributes not explicitly mentioned in the curricula vitae. Thus almost a quarter of all the reasons offered by the decision makers involved the use of irrelevant attributes to confer uniqueness on the chosen alternative. Quite apart from unequal importance weighting of attributes, a form of singleton bias through the use or inference of irrelevant attributes accounts for more than two-thirds of the self-reported reasons for choice.

Apart from the apparent lexicographic ordering of designated attributes or subsets of attributes and the use or inference of non-designated attributes, which together account for more than 88 per cent of the self-reported reasons for choice, Table 6 also indicates other reasons for choice. A number of decision makers claimed that their chosen candidates had more designated qualifications than the other two, although in reality every choice was completely balanced, and some claimed that their chosen candidates had all the designated qualifications, which was never the case; in fact, every candidate had exactly three designated qualifications. Only 7/160 (approximately 4 per cent) of stated reasons fell into these categories, which comprise errors or delusions. Finally, three of the 160 reasons (approximately 2 per cent) amounted to inferring relevant attributes not mentioned in the curricula vitae from information that was mentioned in the curricula vitae. Thus only a small percentage of decision makers behaved in a manner that could be described as obviously irrational.

The results of Experiment 2 reveal that, in all three decision frames (choosing a university, choosing a house/apartment, and choosing an unspecified alternative in an abstract decision frame), the decision makers tended to resolve the choices by the crude 
singleton bias of introducing an unbalancing positional attribute and choosing the alternatives presented in the middle position on the computer screen. The choices were invariably made from displays consisting of three alternatives side by side. As Kleinmuntz and Schkade (1993) have pointed out, neither end position of a row of three items is normally perceived as unique, presumably because there are two ends to a row, whereas the central position has a spatial attribute that is obviously unique. There is empirical evidence to show that decision makers who are forced to choose from sets of indistinguishable alternatives, including identical products on supermarket shelves, cubicles in public toilets, and toilet paper from rows of identical dispensers, tend to be biased toward central rather than peripheral alternatives (Christenfeld, 1995). Christenfeld suggested that this phenomenon might be explained in terms of minimizing mental effort. Whatever the explanation for it, it clearly qualifies as a form of singleton bias, and it works as a tie-breaker even if it is given low priority in the lexicographic ordering of attributes. 


\section{Acknowledgements}

The research reported in this article was supported by Grant No. L122251002 from the Economic and Social Research Council of the U.K. as part of the research program on Economic Beliefs and Behavior. We are indebted to Michael Bacharach for suggesting the line of research reported in this article, to Richard Joiner for writing the computer program used in Experiment 2, to Glen J. Crosier, Alistair Dale, Pamela Davies, Andrew Deans, Camille De-Ath, Natalie Dymoke, Claire Ellaway, Anna Fairhurst, and Mark Gardiner for help with the data collection, and to Ken Binmore, Robin Cubitt, Edmund Chattoe, Richard Day, Diego Gambetta, John Hey, Barbara Malt, and Bob Sugden for comments on earlier versions of this article.

Correspondence concerning this article should be addressed to Andrew M. Colman, Department of Psychology, University of Leicester, Leicester LE1 7RH, England. Electronic mail may be sent via the Internet to amc@leicester.ac.uk. 


\section{References}

Aaker, David A., 1991, Managing brand equity (Free Press, New York).

Carpenter, Gregory S., Glazer, Rashi, and Nakamoto, Kent, 1994, Meaningful brands from meaningless differentiation: The dependence on irrelevant attributes, Journal of Marketing Research 31, 339-350.

Christenfeld, Nicholas, 1995, Choices from identical options, Psychological Science, 6, 5055.

Cohen, Jacob, 1988, Statistical power analysis for the behavioral sciences, 2nd ed. (Erlbaum, Hillsdale, NJ).

Cohen, Jacob, 1992, A power primer, American Psychologist, 112, 155-159.

Dawes, Robyn M., 1979, The robust beauty of improper linear models in decision making, American Psychologist, 34, 571-582.

Day, Richard H., 1971, Rational choice and economic behavior, Theory and Decision, 1, 229-251.

Day, Richard H., 1996, Satisficing multiple preferences in and out of equilibrium, in R. Fabella and E. de Dios (Eds), Choice, growth and development: Emerging and enduring issues, essays in honor of José Encarnación (Quezon City, The Philippines, University of the Philippines Press, pp. 1-23).

Encarnación, José, 1964, A note on lexicographical preferences, Econometrica, 32, 215-217.

Ford, J. Kevin, Schmitt, Neal, Schechtman, Susan L., Hults, Brian M., and Doherty, M. L., 1989, Process tracing methods: Contributions, problems and neglected research issues, Organizational Behavior and Human Decision Processes, 43, 75-117.

Georgescu-Roegen, Nicholas, 1954, Choice, expectations and measurability, Quarterly Journal of Economics, 68, 503-534.

Kleinmuntz, Don N. and Schkade, David A., 1993, Information displays and decision processes, Psychological Science, 4, 221-227. 
Simon, Herbert A., 1957, Models of man: Social and rational (Wiley, New York).

Slovic, Paul, 1975, Choice between equally valued alternatives, Journal of Experimental Psychology: Human Perception and Performance, 1, 280-287.

Slovic, Paul and Lichtenstein, Sarah, 1971, Comparison of Bayesian and regression approaches to the study of information processing in judgment, Organizational Behavior and Human Performance, 23, 86-112.

Tversky, Amos, 1972, Elimination by aspects: A theory of choice, Psychological Review, 79, 281-299.

Tversky, Amos and Sattah, Shmuel, 1979, Preference trees, Psychological Review, 86, 542573.

Tversky, Amos, Sattah, Shmuel, and Slovic, Paul, 1988, Contingent weighting in judgment and choice, Psychological Review, 95, 371-384.

Westenberg, Mirjam R. M. and Koele, Pieter, 1994, Multi-attribute evaluation processes: Methodological and conceptual issues, Acta Psychologica, 87, 65-84. 
Table 1 . Choice probabilities of alternatives $x, y$, and $z$, defined by equally important attributes $\alpha, \beta, \gamma, \delta, \varepsilon$, from a set of available alternatives $A$ under lexicographic choice Choice probability

\begin{tabular}{cccc}
$\begin{array}{c}\text { Attribute } \\
\text { selected first }\end{array}$ & $\begin{array}{c}P(x, A) \\
(\alpha, \gamma, \varepsilon)\end{array}$ & $\begin{array}{c}P(y, A) \\
(\beta, \gamma, \delta)\end{array}$ & $\begin{array}{c}P(z, A) \\
(\beta, \delta, \varepsilon)\end{array}$ \\
\hline$\alpha$ & 1.0 & 0.0 & 0.0 \\
$\beta$ & 0.0 & 0.5 & 0.5 \\
$\gamma$ & 0.5 & 0.5 & 0.0 \\
$\delta$ & 0.0 & 0.5 & 0.5 \\
$\varepsilon$ & 0.5 & 0.0 & 0.5 \\
\hline Overall $P$ & 0.4 & 0.3 & 0.3 \\
\hline
\end{tabular}


Table 2. Design of Experiment 1: Distribution of five designated qualifications among the three candidates $(A, B, C)$ in each of five groups

\begin{tabular}{lllll} 
Group 1 & Group 2 & Group 3 & Group 4 & Group 5 \\
\hline$A: 1,3,5$ & $A: 2,4,1$ & $A: 3,5,2$ & $A: 4,1,3$ & $A: 5,2,4$ \\
$B: 2,3,4$ & $B: 3,4,5$ & $B: 4,5,1$ & $B: 5,1,2$ & $B: 1,2,3$ \\
$C: 2,4,5$ & $C: 3,5,1$ & $C: 4,1,2$ & $C: 5,2,3$ & $C: 1,3,4$ \\
\hline
\end{tabular}


Table 3. Observed and expected choices in five treatment conditions of Experiment 1

\begin{tabular}{crrrr} 
& \multicolumn{3}{c}{ Candidates } & \\
\cline { 2 - 4 } Group & $A$ & $B$ & $C$ & $\chi^{2}(2, n=20)$ \\
\hline 1 & 14 & 6 & 0 & $14.79^{*}$ \\
2 & 2 & 1 & 17 & $24.09^{*}$ \\
3 & 10 & 5 & 5 & 2.50 \\
4 & 8 & 6 & 6 & 0.41 \\
5 & 4 & 8 & 8 & 1.61 \\
\hline Expected & 6.67 & 6.67 & 6.67 & \\
\hline * $p<.001$ & & & &
\end{tabular}


Table 4. Distribution of candidates chosen in relation to designated qualifications in Experiment 1

\section{Group}

Qualifi-

\begin{tabular}{lrrrrrr} 
cation & 1 & 2 & 3 & 4 & 5 & $\Sigma$ \\
\hline 1 & 14 & 19 & 10 & 14 & 16 & 73 \\
2 & 6 & 2 & 15 & 12 & 12 & 47 \\
3 & 20 & 18 & 10 & 14 & 16 & 78 \\
4 & 6 & 3 & 10 & 8 & 12 & 39 \\
5 & 14 & 18 & 15 & 12 & 4 & 63 \\
\hline
\end{tabular}


Table 5. Distribution of candidates chosen in relation to triplets of designated qualifications in Experiment 1

\section{Group}

Qualifi-

\begin{tabular}{lllllll} 
cations & 1 & 2 & 3 & 4 & 5 & $\Sigma$ \\
\hline $1,2,3$ & 0 & 0 & 0 & 0 & 8 & 8 \\
$1,2,4$ & 0 & 2 & 5 & 0 & 0 & 7 \\
$1,2,5$ & 0 & 0 & 0 & 6 & 0 & 6 \\
$1,3,4$ & 0 & 0 & 0 & 8 & 8 & 16 \\
$1,3,5$ & 14 & 17 & 0 & 0 & 0 & 31 \\
$1,4,5$ & 0 & 0 & 5 & 0 & 0 & 5 \\
$2,3,4$ & 6 & 0 & 0 & 0 & 0 & 6 \\
$2,3,5$ & 0 & 0 & 10 & 6 & 0 & 16 \\
$2,4,5$ & 0 & 0 & 0 & 0 & 4 & 4 \\
$3,4,5$ & 0 & 1 & 0 & 0 & 0 & 1 \\
\hline
\end{tabular}


Table 6. Self-reported reasons for choices in Experiment 1

Implicit underweighting of designated attribute(s) (e.g., "Although he has no knowledge of foreign language listed, he has all the other requested qualifications")

Focus on non-designated attribute(s) mentioned on curriculum vitae ("He is the youngest applicant")

Implicit overweighting of 3 specific designated attributes (e.g., "He works for a footwear company already, has previous engineering experience ..., and fluent in other language")

Implicit overweighting of 1 specific designated attribute (e.g., "Most experienced managerial skills as well as fulfilling other criteria")

Implicit overweighting of 2 specific designated attributes (e.g., “Good job experience. He can speak another language")

Inference of non-designated attribute(s) not mentioned in curriculum vitae (e.g., "He does amateur dramatics and so may be an outgoing or friendly person")

Ambiguous or unclassifiable (e.g., "Eastwood is the best fit")

*Chosen candidate supposed to possess more designated attributes 
than the others (e.g., "He fulfils more criteria required than other applicants")

*Chosen candidate supposed to possess all required attributes (e.g., "He fits all of the job requirements")

Inference of designated attribute(s) not mentioned on curriculum vitae (e.g., "He likes travel therefore likely he has some foreign language skills") 3

* The starred reasons were spurious: every candidate possessed three of the five designated attributes. 
Table 7. Typical set of alternatives in choice phase of Experiment 2

House A

Having gas central

heating

Not having a fitted

kitchen

Having a garage

Having a garage

Being cheap to heat

Not being cheap to

heat

Not having a telephone Having a telephone

House C

Not having gas central

heating

Not having a garage

Being cheap to heat

Having a telephone 
Table 8. Counterbalancing of attributes and singletons in Experiment 2 (attributes possessed by alternatives are starred)

Alternative

\begin{tabular}{|c|c|c|c|}
\hline Cell & $A$ & $B$ & $C$ \\
\hline 1 & $1^{*}, 2,3^{*}, 4,5^{*}$ & $1,2^{*}, 3^{*}, 4^{*}, 5$ & $1,2^{*}, 3,4^{*}, 5^{*}$ \\
\hline 2 & $1^{*}, 2^{*}, 3,4^{*}, 5$ & $1,2,3^{*}, 4^{*}, 5^{*}$ & $1^{*}, 2,3^{*}, 4,5^{*}$ \\
\hline 3 & $1,2^{*}, 3^{*}, 4,5^{*}$ & $1^{*}, 2,3,4^{*}, 5^{*}$ & $1^{*}, 2^{*}, 3,4^{*}, 5$ \\
\hline 4 & $1^{*}, 2,3^{*}, 4^{*}, 5$ & $1^{*}, 2^{*}, 3,4,5^{*}$ & $1,2^{*}, 3^{*}, 4,5^{*}$ \\
\hline 5 & $1,2^{*}, 3,4^{*}, 5^{*}$ & $1^{*}, 2^{*}, 3^{*}, 4,5$ & $1^{*}, 2,3^{*}, 4^{*}, 5$ \\
\hline 6 & $1,2^{*}, 3^{*}, 4^{*}, 5$ & $1^{*}, 2,3^{*}, 4,5^{*}$ & $1,2^{*}, 3,4^{*}, 5^{*}$ \\
\hline 7 & $1,2,3^{*}, 4^{*}, 5^{*}$ & $1^{*}, 2^{*}, 3,4^{*}, 5$ & $1^{*}, 2,3^{*}, 4,5^{*}$ \\
\hline 8 & $1^{*}, 2,3,4^{*}, 5^{*}$ & $1,2^{*}, 3^{*}, 4,5^{*}$ & $1^{*}, 2^{*}, 3,4^{*}, 5$ \\
\hline 9 & $1^{*}, 2^{*}, 3,4,5^{*}$ & $1^{*}, 2,3^{*}, 4^{*}, 5$ & $1,2^{*}, 3^{*}, 4,5^{*}$ \\
\hline 10 & $1^{*}, 2^{*}, 3^{*}, 4,5$ & $1,2^{*}, 3,4^{*}, 5^{*}$ & $1^{*}, 2,3^{*}, 4^{*}, 5$ \\
\hline 11 & $1,2^{*}, 3,4^{*}, 5^{*}$ & $1,2^{*}, 3^{*}, 4^{*}, 5$ & $1^{*}, 2,3^{*}, 4,5^{*}$ \\
\hline 12 & $1^{*}, 2,3^{*}, 4,5^{*}$ & $1,2,3^{*}, 4^{*}, 5^{*}$ & $1^{*}, 2^{*}, 3,4^{*}, 5$ \\
\hline 13 & $1^{*}, 2^{*}, 3,4^{*}, 5$ & $1^{*}, 2,3,4^{*}, 5^{*}$ & $1,2^{*}, 3^{*}, 4,5^{*}$ \\
\hline 14 & $1,2^{*}, 3^{*}, 4,5^{*}$ & $1^{*}, 2^{*}, 3,4,5^{*}$ & $1^{*}, 2,3^{*}, 4^{*}, 5$ \\
\hline 15 & $1^{*}, 2,3^{*}, 4^{*}, 5$ & $1^{*}, 2^{*}, 3^{*}, 4,5$ & $1,2^{*}, 3,4^{*}, 5^{*}$ \\
\hline
\end{tabular}


Table 9. Frequencies of singleton and non-singleton choices in Experiment $2(O=$ observed, $E=$ expected)

Decision Frame

\begin{tabular}{|c|c|c|c|c|c|c|}
\hline & \multicolumn{6}{|c|}{ Decision Frame } \\
\hline & \multicolumn{2}{|c|}{ University } & \multicolumn{2}{|c|}{ House/Apartment } & \multicolumn{2}{|c|}{ Abstract } \\
\hline & $O$ & $E$ & $O$ & $E$ & $O$ & $E$ \\
\hline Singleton & 40 & 33.33 & 36 & 33.33 & 38 & 33.33 \\
\hline Non-Singleton & 60 & 66.67 & 64 & 66.67 & 62 & 66.67 \\
\hline
\end{tabular}




\section{Author Note}

Andrew M. Colman is a Reader in psychology at the University of Leicester, U.K., having previously taught at Rhodes and Cape Town Universities. He is the founder and former editor of the journal Current Psychology, and his publications include over 150 articles and a number of books, including Facts, Fallacies and Frauds in Psychology (Routledge, London, 1987), What is Psychology? 3rd ed. (Routledge, London, 1999), Game Theory and its Applications in the Social and Biological Sciences, 2nd ed., (Butterworth-Heinemann, Oxford, 1995). He is the editor of the Companion Encyclopedia of Psychology (Routledge, London, 1994), and of the Longman Essential Psychology Series (Longman, London, 1995).

Jonathan A. Stirk graduated in psychology from the University of Leicester in 1991, where he later worked as a Research Assistant. His publications include "Stackelberg thinking in mixed-motive games", in C. Roland Lévy (Ed.), Social and Economic Representations (1996, vol. 2, pp. 1115-1126), and "Stackelberg reasoning in mixed-motive games: An experimental investigation", (Journal of Economic Psychology, 1998), both coauthored with Andrew M. Colman. 